\title{
Finite Element Modeling and Dynamic Analysis of Rotating Disc and Drum Connected by Bolted Joints
}

\author{
Z. Y. Qin ${ }^{\mathrm{a}}$, H. Y. Wang ${ }^{\mathrm{b}}$ and F. L. Chu ${ }^{\mathrm{c}}$ \\ Department of Precision Instruments and Mechanology, Tsinghua University, Beijing 100084, China \\ a'qinzy@mail.tsinghua.edu.cn, ${ }^{b} a x i a l 888 @ 163 . c o m,{ }^{c}$ chufl@mail.tsinghua.edu.cn
}

Keywords: disc-drum structure, bolted joint, FEM, dynamics

\begin{abstract}
The disc-drum type rotors are commonly used in large gas turbine engines. In this paper, the dynamic characteristics of single stage disc-drum structure are studied based on the finite element model, where the connecting bolts are modeled using solid elements and the frictional contact at the joint interface is accommodated. In order to evaluate the nonlinearity resulting from the bolted joints, a finite element (FE) model fixing the disc and drum together is also established, and the simulation results based on the two models are compared. The effects of the bolt preload and the rotating speed on the dynamic characteristics of the disc-drum structure are also discussed. The works proposed in this paper help to enhance the understanding of the dynamics of the disc-drum type rotor.
\end{abstract}

\section{Introduction}

The disc-drum type rotors are commonly employed in large gas turbine engines, where the drums are used to connect the adjacent discs through the welded and bolted joints and transmit loads [1], as shown in Fig. 1. Due to the plate and shell characteristics and the local nonlinearity caused by the bolted joints, the disc-drum structure tends to vibrate with complex dynamic behaviors. The vibration of the disc-drum structure may lead to rub and impact between the rotor and the stator, which affects the rotor dynamics and even leads to structure damage. Hence, it is of great importance to investigate the dynamic characteristics of the disc-drum structure, which will help to improve the performance and reliability of the gas turbine engines comprising the disc-drum type rotors.

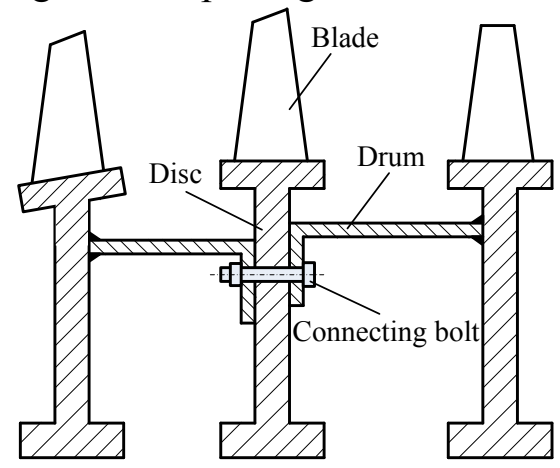

Fig. 1. Sketch of the disc-drum structure

Much research has been carried out to study the dynamics of the disc-drum type rotors. However, in these studies the rotors were usually simplified as beams, where the plate and shell characteristics of the disc and drum were neglected [2-4]. In order to overcome the shortages of the beam model, some researchers employed the three-dimensional (3D) solid elements to model complex rotors based on the FEM. Rao [5] proposed a 3D FE model to study the dynamics of the three-level rotor system including the inner rotor, the outer rotor and the flexible casing, where the advantages of the 3D FE model compared with the $1 \mathrm{D}$ beam model were discussed. Ref. [6] constructed the 3D FE model for the disc-rotor and investigated the dynamic characteristics of the rotor. In all the rotor models mentioned above, the rotors were considered to be continuous and the nonlinear joint interfaces were not considered. Actually, various types of joints, such as the bolted joint, the welded joint, the spline joint and so on, are commonly adopted in the large rotors and may affect the rotor dynamics. However, little research was performed on the nonlinear behavior of the 
joints in rotor structures. Ref. [7] studied the dynamical state of the typical joint structures in the aero-engine rotor. The works in the literature were focused on the variation of the stiffness and the contact state with respect to the external load and the structure geometry, whereas the effect of the nonlinear joint behavior on the dynamics of the rotor was not analyzed in detail.

In this paper, the dynamic characteristics of the single stage disc-drum structure are studied considering the plate and shell characteristics and the nonlinear local joint behavior. Firstly, the nonlinear finite element model for the disc-drum structure is established, where the connecting bolts are modeled and the frictional contact of the joint components is accommodated. Then, simulations are carried out to study the dynamic characteristics of the disc-drum structure. And the simulation results are compared with those based on the disc-drum model without considering the nonlinear joint between the disc and the drum. Finally, parametric studies are carried out to investigate how physical parameters, such as the bolt preload and the rotating speed, affect the dynamic characteristics of the disc-drum structure.

\section{Finite element modeling}

The last stage disc-drum structure from the compressor of a certain type aero-engine is investigated here. The diameter of the drum middle surface is $270 \mathrm{~mm}$, the thickness of the drum is $2.5 \mathrm{~mm}$, and 30 bolts are distributed uniformly in the circumference to connect the disc and drum. The disc and drum are fabricated from the same alloy, i.e. TC4, whose density is $4420 \mathrm{~kg} / \mathrm{m}^{3}$, elastic modulus $E$ is $107 \mathrm{GPa}$, and the Poisson's ratio $v$ is 0.3 . There exists frictional contact between the joint components, where the friction coefficient at the contact surfaces is set to be 0.1 .

The FE model of the disc-drum structure is illustrated in Fig. 2, where the disc, drum and bolts are modeled using the brick elements SOLID 45. Since the main purpose of this paper is to analyze the dynamics of the disc-drum structure, the blades mounted on the disc are neglected to simplify the analysis procedure. It should be noted that the disc-drum structure is cyclic symmetric, cyclic model can be adopted to improve the computational efficiency. However, cyclic symmetric analysis may lead to the loss of certain modes [8]. Hence, a full model is constructed here, which contains 86670 nodes and 74280 elements. In this model, frictional surface-to-surface contact is defined between each contact pair using contact elements CONTA173 and target segment elements TARGE170.
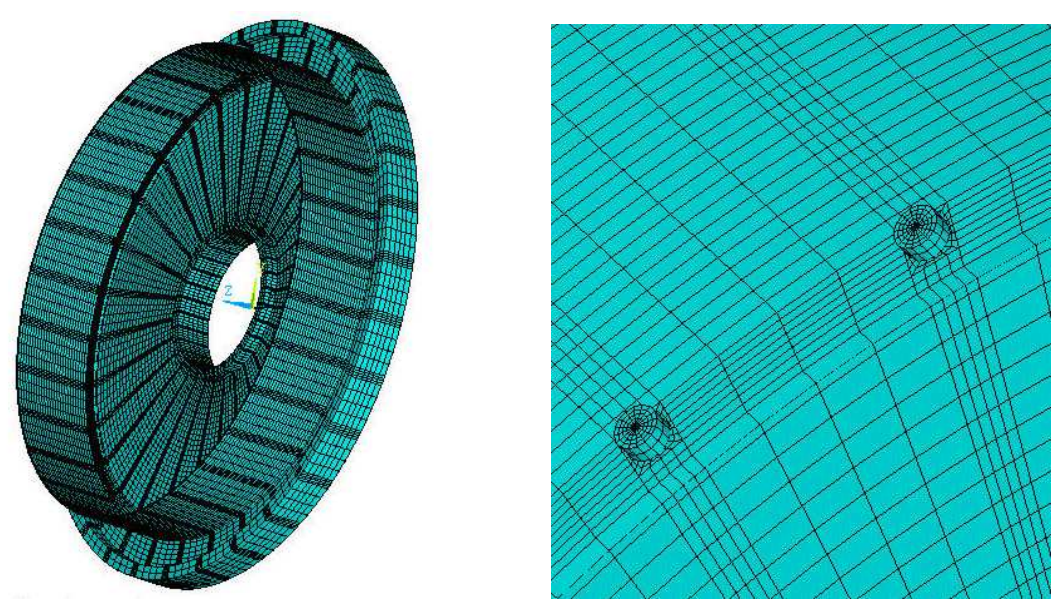

Fig. 2. FE model for the disc-drum structure.

Considering the facts that the disc-drum structure studied here is the last stage and that the drum is connected to the adjacent stage disc through welding, the nodes of the disc are free and all degrees of freedom (dofs) of the nodes on the drum welding surface are constrained. In order to take into account the effects of nonlinear contact and the rotating state, the modal analysis is carried out in three steps as follows: first prestressing the connecting bolts using the virtual thermal deformation method in the static analysis; then applying the rotating speed on the disc-drum structure; finally performing the prestressed modal analysis. 
In order to evaluate the effects of the nonlinear joint interface on the dynamic characteristics of the disc-drum structure, another FE model for the disc-drum structure is also constructed, where the disc and drum are fixed together without considering the bolted joints. For convenience, this model is referred to as the fixed model and the model consisting of the bolted joints is referred to as the joint model.

\section{Simulation results and discussions}

The preload of $5 \mathrm{kN}$ and the rotating speed of $3000 \mathrm{r} / \mathrm{min}$ are applied to the FE model, the first 8 mode shapes of the joint model are calculated and shown in Fig. 3, where it can be seen that the mode shapes of the disc-drum structure are the combination of those of the disc and drum, which are dominated by the disc mode shapes for the lower modes. The mode shapes calculated from the fixed model coincide with those of the joint model.

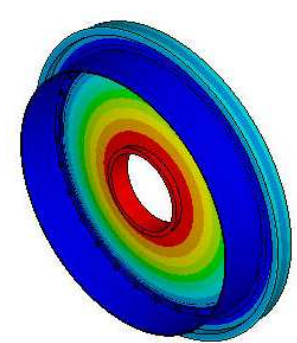

(a) first mode

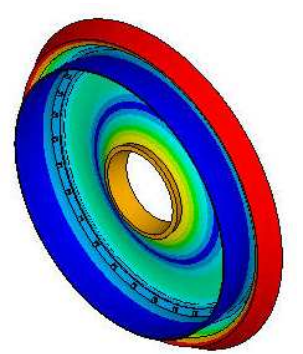

(a) fifth mode

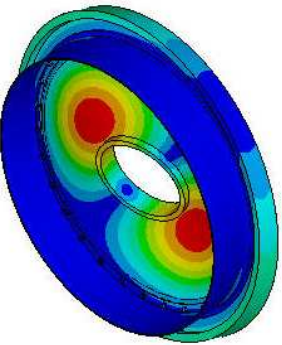

(b) second mode

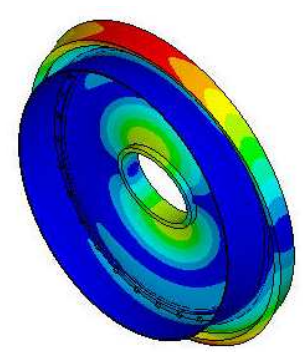

(b) sixth mode

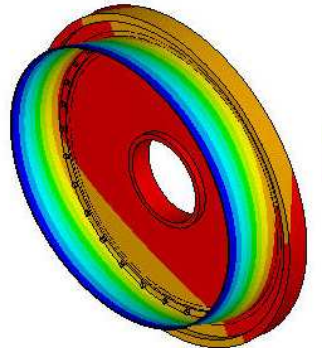

(c) third mode

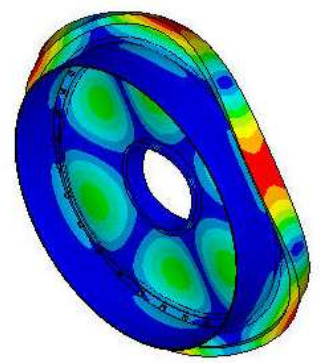

(c) seventh mode

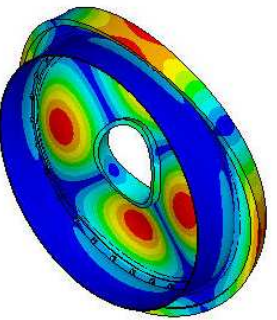

(d) fourth mode

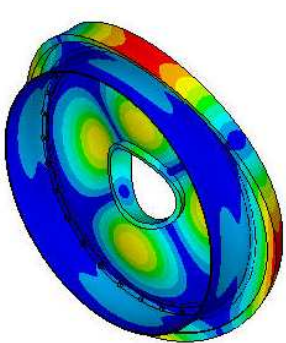

(d) eighth mode

Fig. 3. The first 8 modal shapes of the disc-drum structure

The resonant frequencies of the first 8 mode calculated from the joint model and the fixed model are compared in Table 1. It is indicated that the resonant frequencies of the disc-drum structure considering the joint interfaces are smaller than those neglecting the bolted joints, which means that the bolted joints decrease the stiffness of the structure. It is also revealed that the deviation between the resonant frequencies based on the two models gets a little larger for higher mode, except for the third mode, of which the deviation is obviously larger than that of the fourth mode. By referring to Fig. 3 , the third mode shape represents the bending characteristics of the drum. Hence, it can be concluded that the joint behavior affects the bending modes more significantly.

Table 1 Comparison of the resonant frequencies calculated from the joint and fixed models

\begin{tabular}{cccc}
\hline mode order & joint model $[\mathrm{Hz}]$ & fixed mode $[\mathrm{Hz}]$ & Deviation [\%] \\
\hline 1 & 306.73 & 308.54 & 0.59 \\
2 & 584.73 & 587.62 & 0.49 \\
3 & 959.26 & 979.76 & 2.09 \\
4 & 979.27 & 992.00 & 1.28 \\
5 & 1181.0 & 1199.9 & 1.58 \\
6 & 1323.1 & 1357.3 & 2.52 \\
7 & 1341.5 & 1373.6 & 2.34 \\
8 & 1560.1 & 1605.2 & 2.81 \\
\hline
\end{tabular}


Maintaining the rotating speed of $3000 \mathrm{r} / \mathrm{min}$ unchanged, different preload of $1 \mathrm{kN}, 10 \mathrm{kN}, 20 \mathrm{kN}$ and $30 \mathrm{kN}$ are applied to the joint model. The resonant frequencies corresponding to the first 8 modes under different rotating speed are listed in Table 2. Comparing the simulation results under different preload indicates that along with the increment of the magnitude of the preload, the resonant frequencies take place small but definite growth for relatively small preload. For the large preload, the resonant frequencies change little as the preload value increases.

Table 2 Comparison of the resonant frequencies under different preload

\begin{tabular}{ccccc}
\hline mode order & $\begin{array}{c}\text { preload of } 1 \mathrm{kN} \\
{[\mathrm{Hz}]}\end{array}$ & $\begin{array}{c}\text { preload of 10kN } \\
{[\mathrm{Hz}]}\end{array}$ & $\begin{array}{c}\text { preload of 20kN } \\
{[\mathrm{Hz}]}\end{array}$ & $\begin{array}{c}\text { preload of 30kN } \\
{[\mathrm{Hz}]}\end{array}$ \\
\hline 1 & 305.67 & 306.72 & 306.60 & 306.39 \\
2 & 583.03 & 584.73 & 584.60 & 584.39 \\
3 & 906.63 & 959.32 & 959.37 & 959.42 \\
4 & 973.85 & 979.26 & 979.11 & 978.85 \\
5 & 1174.1 & 1180.9 & 1180.7 & 1180.5 \\
6 & 1311.1 & 1323.0 & 1322.9 & 1322.8 \\
7 & 1329.8 & 1341.5 & 1341.4 & 1341.2 \\
8 & 1473.2 & 1560.1 & 1560.1 & 1560.2 \\
\hline
\end{tabular}

The resonant frequencies of the first 4 modes of disc-drum structure under the preload of $5 \mathrm{kN}$ and different rotating speed of $0,2000 \mathrm{r} / \mathrm{min}, 4000 \mathrm{r} / \mathrm{min}, 6000 \mathrm{r} / \mathrm{min}$ and $8000 \mathrm{r} / \mathrm{min}$ are illustrated in Fig. 4, where it can be observed that the variation trends of the resonant frequencies calculated from the joint model and the fixed model are consistent, that is, the resonant frequencies becomes large with the increment of the rotating speed except for that of the third mode. The resonant frequency of the third mode, i.e. the bending mode, changes little as the rotating speed increases, which informs that the rotating state has little effect on the bending mode.

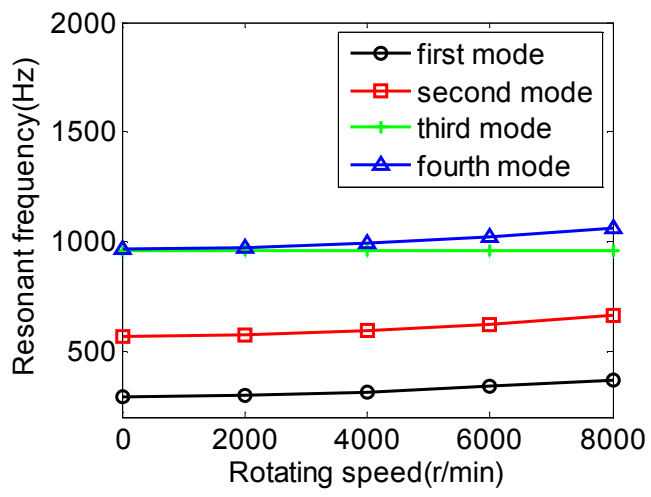

(a) Joint model

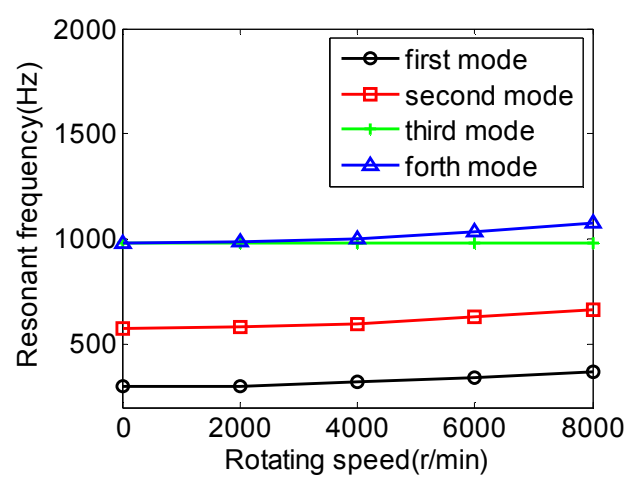

(b) fixed model

Fig. 4. Variation of resonant frequencies with respect to rotating speed

\section{Conclusions}

The FE model of the disc-drum structure was developed considering the bolted joints between the disc and drum. The dynamics of the disc-drum structure were investigated, where the effects of the bolt preload and the rotating speed were evaluated.

It was revealed from the simulation results that the bolted joint decreased the stiffness of the combined structure and affected the bending modes more significantly. Increasing the joint preload improved the structure stiffness. However, the effect is not significant especially for the relatively large preload conditions. Most of the resonant frequencies of the disc-drum structure increase along with the increment of the rotating speed, whereas the resonant frequency of the bending mode changes little. 
It can be concluded that the effect of the rotating speed on the dynamics of the disc-drum structure is obvious and should be considered, whereas the effect of the bolted joints can be neglected for the cases, where the joint preload is relatively large and the dynamics are dominated by the relatively lower modes.

\section{References}

[1] Editorial Board of Aero-engine Design Manual. Aero-engine design manual (19th Part): rotor dynamics and whole engine vibration. Beijing: Aviation Industry Press, 2000. (in Chinese)

[2] H.W.D. Chiang, C.N. Hsu, S.H. Tu. Rotor-bearing analysis for turbomachinery single- and dual-rotor systems, J. Propul. Power. 20 (2004) 1096-1104.

[3] G. Chen. A new rotor-ball bearing-stator coupling dynamics model for whole aero-engine vibration, ASME J. Vib. Acoust.. 131 (2009) 0610091-0610099.

[4] W.Q. Deng, F.Y. Guo, D.P. Gao. Dynamic characteristics calculation for a high speed flexible rotor of an aero-engine, Journal of Vibration and Shock. 25 (2006) 130-133. (in Chinese)

[5] J.S. Rao, R. Sreenivas. Dynamic of a three level rotor system using solid elements, Proceedings of ASME Turbo Expo 2003, Power for Land, Sea, and Air. 2003 1-6.

[6] M.J. Lu, H.P. Geng, B.S. Yang, L. Yu. Finite element method for disc-rotor dynamic characteristics analysis of gas turbine rotor considering contact effects and rod preload, Proceedings of the 2010 IEEE International Conference on Mechatronics and Automation. 2010 1179-1183.

[7] S.G. Liu, Y.H. Ma, J. Hong. Studies on dynamical state stability of the aero-engine rotor joint structures, The 8th IFToMM International Conference on Rotor Dynamics. 2010 214-221.

[8] Y. Song, Y.Q. Ai, B. Liang. Mastery of ANSYS 7.0 finite element analysis, Tsinghua university press, Beijing, 2003. (in Chinese) 